\title{
Stochastic Resonance: a Comparative Study of Two-State Models
}

\author{
Peter Imkeller and Ilya Pavlyukevich
}

\begin{abstract}
We consider a dynamical system describing the motion of a particle in a double well potential with a periodic perturbation of very small frequency, and an additive stochastic perturbation of amplitude $\varepsilon$. It is in stochastic resonance if the solution trajectories amplify the small periodic perturbation in a 'best possible way'. Systems of this type first appeared in simple energy balance models designed for a qualitative explanation of global glacial cycles. Large deviations theory provides a lower bound for the proportion of the amplitude and the logarithm of the period above which quasi-deterministic periodic behavior can be observed. However, to obtain optimality, one has to measure periodicity with a measure of quality of tuning such as spectral power amplification favored in the physical literature. In a situation where the potential switches discontinuously between two spatially antisymmetric double well states we encounter a surprise. Contrary to physical intuition, the stochastic resonance pattern is not correctly given by the reduced dynamics described by a two state Markov chain with periodic hopping rates between the potential minima which mimic the large (spatial) scale motion of the diffusion. Only if small scale fluctuations inside the potential wells where the diffusion spends most of its time are carefully eliminated, the Markov chain gives the correct picture.
\end{abstract}

\section{Background and paradigm}

The paradigm of stochastic resonance (SR) emerged from papers by C. Nicolis [15] and Benzi et al. [2, 3, 4] which were devoted to the mathematical explanation of the phenomenon of glacial cycles. The model they created is based on the following observations. Modern measurement techniques allow to determine concentrations of an oxygen isotope in deep sea core sediments which in turn provide rough estimates of the global mean temperature of the earth at the time they were deposited. This way at least seven changes between 'cold' and 'warm' periods were

Received by the editors October 15, 2002.

2000 Mathematics Subject Classification. Primary 60H10; Secondary 60J60, 60J27, 86A04, 35P15, 37A30, 37N10.

Key words and phrases. Stochastic resonance, spectral gap, stochastic differential equation, energy balance model, potential diffusion, effective dynamics. 
detected during approximately the last 700,000 years. They occur abruptly and with roughly the same period of about $10^{5}$ years. The quoted papers aimed at suggesting a simple mathematical model to account for this deterministic-looking periodicity.

The proposed model just appeals to conservation of radiative energy and supposes that the earth's temperature $T$ satisfies a simple energy-balance equation (for an extended review see [11]), i.e. the instant change of the global temperature is proportional to the difference between incoming and outgoing radiative energy:

$$
c \frac{d T(t)}{d t}=Q(t)(1-a(T(t)))-\sigma T(t)^{4}, \quad c>0 .
$$

In the simplest case considered here it is assumed that the total energy flux emitted by the earth is given by the Stefan-Boltzmann law which in fact is valid only for a black body radiator.

The absorbed energy depends on two factors. The global solar function $Q(t)$ describes the flux of the solar energy which reaches the earth at time $t$. Assuming that the solar activity is a constant $Q_{0}$, the function $Q$ depends on the distance between the earth and the sun as well as on the inclination of the earth's axis, and due to the gravitational influence of Jupiter exhibits a slow periodic variation of a period of about $10^{5}$ years. The variation is estimated to be $0.1 \%$ of $Q_{0}$. Thus we put

$$
Q(t)=Q_{0}-b \sin \omega t, \quad b \approx 0.001 Q_{0}, \quad \omega \approx\left(2 \pi 10^{5}\right)^{-1}\left[\mathrm{yr}^{-1}\right] .
$$

On the other hand, not all the solar radiation reaching the atmosphere is absorbed: the proportion of absorbed radiation is determined by the earth's albedo $a$ which depends locally on the earth's average surface temperature $T$. The simple albedo model used here appears in the papers by Budyko [5] and Sellers [18], see Fig. 1 (1.). $a(T)$ is chosen to be a constant close to 1 for low temperatures $T \leq \underline{T}$. In this
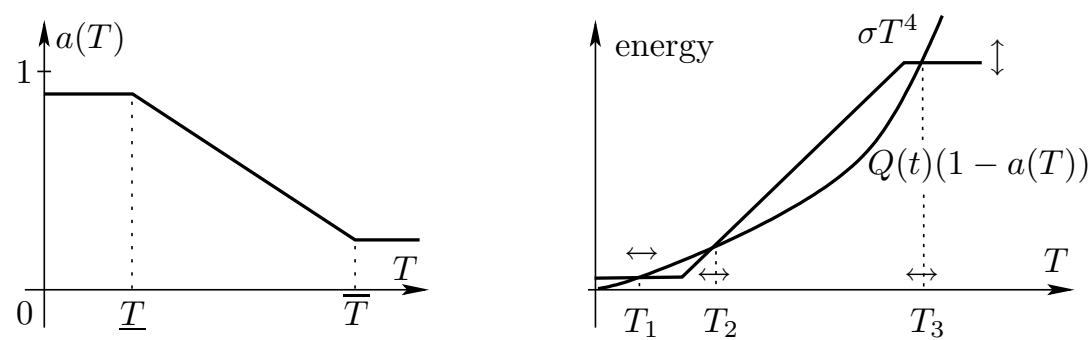

Fig. 1. Earth's albedo (l.) and incoming and outgoing radiative energies (r.).

temperature regime all surface water is supposed to be frozen and to cover a big portion of the planet by a bright ice layer making the reflection ratio relatively high. For high temperatures $T \geq \bar{T}$ the green-brown vegetation results in a low 
reflection ratio. In the regime between $\underline{T}$ and $\bar{T}$ the function $a(T)$ is interpolated linearly.

Thus, the right hand side of (1.1) is a difference of two functions, see Fig. 1 (r.). For appropriate values of parameters the dynamical system (1.1) has two metastable equilibrium states $T_{1}$ and $T_{3}$ separated by the unstable state $T_{2}$. The lower metastable state $T_{1}$ is interpreted as describing ice age temparatures whereas $T_{3}$ determines warm ages.

This model of climate has major shortcomings and therefore cannot picture reality. Indeed, solutions of (1.1) converge to either $T_{1}$ or $T_{3}$ and oscillate with periods of $10^{5}$ years with relatively small amplitudes, due to the smallness of $b$. Most importantly, however, the typically observed spontaneous and rapid transitions between 'cold' and 'warm' states are impossible.

To overcome this difficulty C. Nicolis and Benzi et al. added a noise term to the energy-balance equation (1.1) and obtained the following simple SDE for the global temperature:

$$
c \frac{d T(t)}{d t}=Q(t)(1-a(T(t)))-\sigma T(t)^{4}+\sqrt{\varepsilon} \dot{W}_{t}, \quad c>0,
$$

where $W$ is a standard one-dimensional Brownian motion and $\varepsilon>0$. In this setting, transitions between meta-stable states become possible, and - most importantly - transition times are small (of the order $10^{2}$ years) and much more realistic.

In the one-dimensional setting of the system (1.2) one can always represent the drift term as a gradient in the variable $T$ of some potential function $U$, i.e. we can find $U$ such that

$$
-\frac{\partial U(T, t)}{\partial T}=Q(t)(1-a(T(t)))-\sigma T(t)^{4}
$$

Of course, the potential depends on time. Fig. 2 shows the incoming and outgoing radiation, their difference, and the corresponding potential function at times when the solar constant takes its minimum (left column) and maximum (right column). In terms of the potential $U$, equation (1.2) describes the dynamics of an overdamped Brownian particle in a double well potential, where the minima of the potential wells correspond to the 'cold' and 'warm' global temperatures. The depths of the potential wells vary periodically in time, and the left well is deeper for approximately $5 \times 10^{4}$ years. Clearly, this periodic and deterministic variation of the wells' depths is the most important feature of the potential $U$.

To catch the essentials of the effect and at the same time to simplify the problem we will work with a time-space asymmetric double well potential. In the strip $(x, t) \in \mathbb{R} \times[0,1)$ it is defined by the formula

$$
U(x, t)= \begin{cases}U_{1}(x), & t \in\left[0, \frac{1}{2}\right), \\ U_{2}(x)=U_{1}(-x), & t \in\left[\frac{1}{2}, 1\right) .\end{cases}
$$

It is periodically extended for all times $t$ by the relation $U(\cdot, t)=U(\cdot, t+1)$, see Fig. 3. We assume that the potential has two local minima at \pm 1 and a local maximum at 0 , that $U_{1}(-1)=-\frac{V}{2}, U_{1}(1)=-\frac{v}{2}, \frac{2}{3}<\frac{v}{V}<1$, and $U_{1}(0)=0$. We 

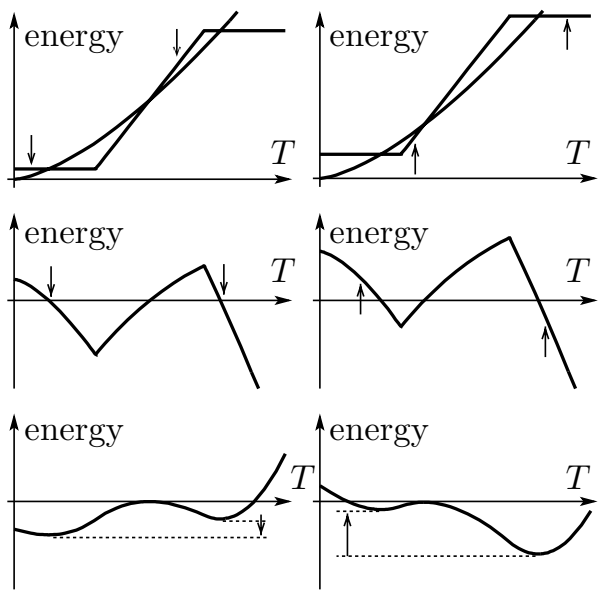

Fig. 2. The drifts of (1.2) and the corresponding potentials at times when the solar constant takes its minimum (l.) and maxi$\operatorname{mum}(\mathrm{r}$.$) .$
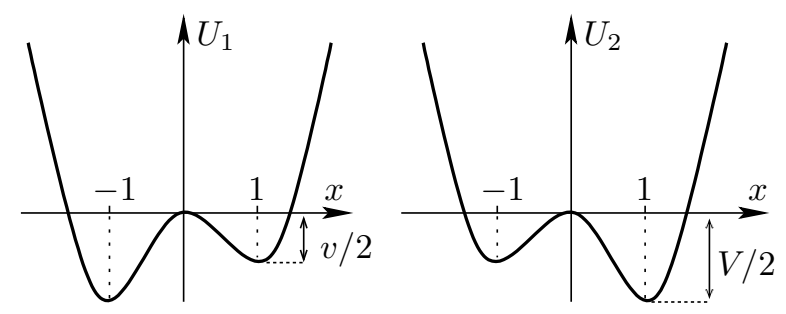

Fig. 3. Time-periodic potential $U$.

also suppose that the extrema of $U$ are not degenerate, i.e. the curvatures at these points do not vanish.

A trajectory of a Brownian particle in this potential is described by the SDE

$$
d X_{t}^{\varepsilon, T}=-U^{\prime}\left(X_{t}^{\varepsilon, T}, \frac{t}{T}\right) d t+\sqrt{\varepsilon} d W_{t}, \quad X_{0}^{\varepsilon, T}=x \in \mathbb{R}
$$

where $\varepsilon>0$ is the noise intensity, and $T>0$ the period.

The problem of finding an intensity characterizing stochastic resonance now consists in determining an optimal tuning $\varepsilon=\varepsilon(T)$, i.e. the noise intensity for which the trajectories $X^{\varepsilon, T}$ look 'as periodic as possible'. Of course, in these terms stochastic resonance is a rather unprecise concept. To make it precise at least requires measuring periodicity in diffusion trajectories. 


\section{Freidlin's approach}

Using large deviations theory, M. Freidlin [8] explains periodicity as a quasideterministic property of diffusion trajectories for very large period lengths.

Consider, for example, a Brownian particle in the time homogeneous double well potential $U_{1}$ described by the SDE

$$
d X_{t}^{\varepsilon}=-U_{1}^{\prime}\left(X_{t}^{\varepsilon}\right) d t+\sqrt{\varepsilon} d W_{t} .
$$

For small $\varepsilon$, this stochastic system can be considered as a small white noise perturbation of the deterministic dynamical system $\dot{x}=-U_{1}^{\prime}(x)$. The Freidlin-Wentzell theory of large deviations [9] allows to study asymptotic properties of (2.1) as $\varepsilon \rightarrow 0$ in terms of the geometric properties of the potential $U_{1}$. It is intuitively clear that for small noise intensities the sample paths of (2.1) spend most of the time in small neighborhoods of the meta-stable states \pm 1 . Jumps between the wells occur, but very rarely. The probability of these transitions can be estimated in terms of the so-called quasipotential which measures the work to be done by the diffusion in order to travel between points in the potential landscape. Let, for instance, $X_{0}^{\varepsilon}=-1$ and $x$ belong to the left well. Then the quasipotential $V(-1, x)$ can be found explicitly and equals $2\left(U_{1}(x)-U_{1}(-1)\right)$ and thus twice the height of the potential barrier between -1 and $x$. If $x$ belongs to the right well and $0<x \leq 1$, then $V(-1, x)=2\left(U_{1}(0)-U_{1}(-1)\right)$. Only the way 'up' in the potential landscape contributes to the quasipotential; the way 'down' requires no work and is free. Quasipotentials are defined for rather general classes of stochastic systems by means of action functionals, for details see [9].

Let us define the first entrance time

$$
\tau_{y}^{\varepsilon}=\inf \left\{t \geq 0: X_{t}^{\varepsilon}=y\right\}
$$

Then the quasipotential at $x$ and $y$ determines the exponential order of $\tau_{y}^{\varepsilon}$ if the diffusion starts in $x$ (under the law $\mathbf{P}_{x}$ ) in the limit of small noise, see $[9,8]$.

Theorem 2.1 ('transition law'). For all $\delta>0$ the following holds:

$$
\lim _{\varepsilon \downarrow 0} \mathbf{P}_{x}\left(e^{\frac{1}{\varepsilon}(V(x, y)-\delta)}<\tau_{y}^{\varepsilon}<e^{\frac{1}{\varepsilon}(V(x, y)+\delta)}\right)=1 .
$$

The most important statement of the theorem is that the system (2.1) has two exponentially different intrinsic time scales: the exit time from the left well is of the order $e^{V / \varepsilon}$ whereas the exit time from the right well of the order $e^{v / \varepsilon}$. This results in the following observation: if we consider the trajectories of (2.1) on the exponentially long time intervals $T_{\varepsilon} \propto e^{\lambda / \varepsilon}$, then for $0<\lambda<v$ the trajectory typically does not leave its initial well, and for $\lambda>v$ it spends most of its time near -1 (in probability). In other words, on the different time scales the system (2.1) has different meta-stable states.

This description of meta-stable behaviour can be transferred to the time inhomogeneous system (1.4). Let the period $T=T_{\varepsilon}$ be such that $\lim _{\varepsilon \downarrow 0} \varepsilon \log T_{\varepsilon}=$ $\lambda>0$. Then for $\lambda<v$ the diffusion does not have enough time to leave even the 
shallow well during one half period, and therefore, as in the time homogeneous case, does not leave its initial well. However, if $\lambda>v$, a new effect appears.

Theorem 2.2 (Freidlin, [8]). Let the process $X^{\varepsilon, T}$ satisfy (1.4), and

$$
\lim _{\varepsilon \downarrow 0} \varepsilon \log T_{\varepsilon}>v .
$$

Then for all $A>0$ and $\delta>0$ the following holds true:

$$
\Lambda\left\{t \in[0, A]:\left|X_{T_{\varepsilon} t}^{\varepsilon, T}-\phi(t)\right|>\delta\right\} \rightarrow 0
$$

in $\mathbf{P}_{x}$-probability as $\varepsilon \rightarrow 0$, where $\Lambda\{\cdot\}$ denotes Lebesgue measure on $\mathbb{R}$, and

$$
\phi(t)= \begin{cases}-1, & t(\bmod 1) \in\left[0, \frac{1}{2}\right), \\ 1, & t(\bmod 1) \in\left[\frac{1}{2}, 1\right),\end{cases}
$$

is the coordinate of the global minimum of $U(\cdot, t)$, see Fig. 4.
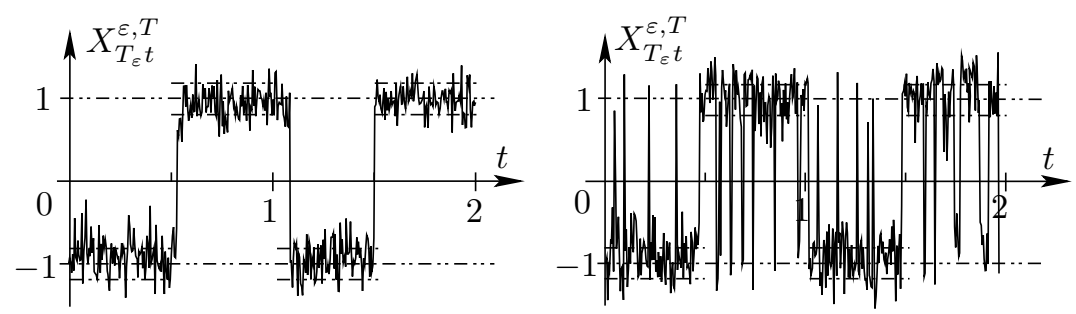

Fig. 4. On time intervals satisfying condition (2.2) the diffusion $X^{\varepsilon, T}$ is close to the deterministic periodic function $\phi$.

Theorem 2.2 suggests a measure of periodicity of diffusion trajectories: take the Lebesgue measure of those times the trajectories spend outside of a $\delta$-tube around the deterministic discontinuous periodic function $\phi$. Condition (2.2) on period $T_{\varepsilon}$ and noise intensity $\varepsilon$ provides a family of tunings, without, however, suggesting an optimal one to determine the resonance point. This is illustrated by Fig. 4 (r.) which clearly suggests that for large $T_{\varepsilon}$ excursions to the 'wrong' well are not very long but frequent and destroy a periodic picture.

\section{Spectral power amplification}

The coefficient of spectral power amplification (SPA) is one of the physicists' favorite measures to measure periodicity of random trajectories, see e.g. [4, 14, 10, 1, 19]. For the diffusion (1.4) it is defined by

$$
\eta^{X}(\varepsilon, T)=\left|\int_{0}^{1} \mathbf{E}_{\mu}\left(X_{T s}^{\varepsilon, T}\right) \cdot e^{2 \pi i s} d s\right|^{2} .
$$


The function $\eta^{X}$ having noise intensity and the period of time variation of the potential as arguments has a clear physical meaning. It shows how much energy is carried by the averaged path of the diffusion with noise amplitude $\varepsilon$ on the frequency $\frac{2 \pi}{T}$. The expectation $\mathbf{E}_{\mu}$ indicates that averages are taken with respect to the time-periodic equilibrium measure of $X^{\varepsilon, T}$. This will be explained in detail later.

Fig. 5 borrowed from [1] where $\Omega$ corresponds to our $\frac{2 \pi}{T}$ and $D$ to the diffusion intensity $\varepsilon$ shows that physicists expect a local maximum of the function $\varepsilon \mapsto \eta^{X}(\varepsilon, \cdot)$. The random paths have their strongest periodic component at the value of $\varepsilon$ for which the maximum is taken. In fact, Fig. 5 depicts not the SPA coefficient of the diffusion itself, but of its so-called 'effective dynamics'. This 'effective dynamics' of the diffusion in a double well potential is a two-state Markov chain living in \pm 1 which reflects only the interwell dynamics of the diffusion while it neglects fluctuations inside the wells. It is a priori believed in the physical literature that the 'effective dynamics' adequately describes the properties of the diffusion in the limit of small noise.

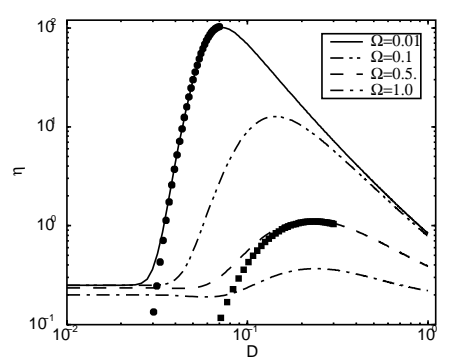

Fig. 5. SPA coefficient as a function of noise amplitude has a well pronounced maximum depending at the frequency of periodic perturbation [1].

To determine the 'optimal tuning' or stochastic resonance point if periodic tuning is measured by SPA means to find the argument $\varepsilon=\varepsilon(T)$ of a local maximum of $\varepsilon \mapsto \eta^{X}(\varepsilon, \cdot)$.

The key to the solution of this problem lies in determining the time-dependent invariant density $\mu$ of $\left(X_{T t}^{\varepsilon, T}\right)_{t \geq 0}$. From now on we follow [17] and [13]. Although the diffusion is not time homogeneous, by enlarging its state space we can consider a two-dimensional time homogeneous Markov process $\left(X_{T t}^{\varepsilon, T}, t(\bmod 1)\right)$ which possesses an invariant law in the usual sense. By definition we identify the timedependent equilibrium density $\mu$ of $\left(X_{T t}^{\varepsilon, T}\right)_{t \geq 0}$ with the invariant density of the two-dimensional process. Indeed, with respect to $\mu$ and for fixed $t$, the law of the real random variable $X_{T t}^{\varepsilon, T}$ has the density $\mu(\cdot, t(\bmod 1))$. The invariant density $\mu$ is a positive solution of the forward Kolmogorov (Fokker-Planck) equation 
$A_{\varepsilon, T}^{*} \mu=0$, where

$$
A_{\varepsilon, T}^{*} \cdot=-\frac{1}{T} \frac{\partial}{\partial t} \cdot+\frac{\varepsilon}{2} \frac{\partial^{2}}{\partial x^{2}} \cdot+\frac{\partial}{\partial x}\left(\cdot \frac{\partial}{\partial x} U\right)
$$

is the formal adjoint of the infinitesimal generator of the two-dimensional diffusion. Moreover, from the time periodicity and time-space antisymmetry of the potential $U(1.3)$ one concludes that $\mu(x, t)=\mu\left(-x, t+\frac{1}{2}\right)$ and $\mu(x, t)=\mu(x, t+1),(x, t) \in$ $\mathbb{R} \times \mathbb{R}_{+}$

This results in the following boundary-value problem used to determine $\mu$. It is enough to solve the Fokker-Planck equation $A_{\varepsilon, T}^{*} \mu=0$ in the strip $(x, t) \in$ $\mathbb{R} \times\left[0, \frac{1}{2}\right]$ with boundary condition $\mu(x, 0)=\mu\left(-x, \frac{1}{2}\right), x \in \mathbb{R}$.

\section{The spectral gap}

We have assumed in (1.3) that the time dependent potential $U$ is a step function of the time variable. In the region $(x, t) \in \mathbb{R} \times\left(0, \frac{1}{2}\right)$ it is identical to a time independent double well potential $U_{1}$, and therefore the Fokker-Planck equation turns into a one-dimensional parabolic PDE

$$
\frac{1}{T} \frac{\partial}{\partial t} \mu(x, t)=\frac{\varepsilon}{2} \frac{\partial^{2}}{\partial x^{2}} \mu(x, t)+\frac{\partial}{\partial x}\left(\mu(x, t) \frac{\partial}{\partial x} U_{1}(x)\right) .
$$

Let $L_{\varepsilon}^{*}$ denote the second order differential operator appearing on the right hand side of (4.1).

To determine $\mu$ we shall use the Fourier method of separation of variables which consists in expanding the solution of (4.1) into a Fourier series with respect to the system of eigenfunctions of the operator $L_{\varepsilon}^{*}$. It turns out that under the condition that $U_{1}$ is smooth and increases 'fast enough' at infinity (for example, as fast as $\left.x^{4}\right)$, the operator $L_{\varepsilon}^{*}$ is essentially self-adjoint in $\mathcal{L}^{2}\left(\mathbb{R}, e^{\frac{2 U_{1}}{\varepsilon}} d x\right)$, its spectrum is discrete and non-positive, and the corresponding eigenspaces are one-dimensional. Denoting by $\|\cdot\|$ and $\langle\cdot, \cdot\rangle$ the norm and the inner product in $\mathcal{L}^{2}\left(\mathbb{R}, e^{\frac{2 U_{1}}{\varepsilon}} d x\right)$ we consider the following formal Floquet type expansion

$$
\mu(x, t)=\sum_{k=0}^{\infty} a_{k} \frac{\Psi_{k}(x)}{\left\|\Psi_{k}\right\|} e^{-T \lambda_{k} t}, \quad(x, t) \in \mathbb{R} \times\left[0, \frac{1}{2}\right],
$$

where $\left\{-\lambda_{k}, \| \frac{\Psi_{k}}{\Psi_{k} \|}\right\}_{k \geq 0}$ is the orthonormal basis corresponding to the spectral decomposition of $L_{\varepsilon}^{*}$, where $\lambda_{0}<\lambda_{1}<\lambda_{2}<\cdots$, and the Fourier coefficients $a_{k}$ are obtained from the boundary condition $\mu(x, 0)=\mu\left(-x, \frac{1}{2}\right), x \in \mathbb{R}$.

Here is the key observation opening the route towards finding local maxima of the SPA coefficient. The terms in the sum (4.2) decay in time exponentially fast with rates $\lambda_{k}$, and therefore the terms corresponding to larger eigenvalues contribute less than the ones belonging to the low lying eigenvalues. This underlines their key importance. Fortunately, in the case of a double well potential the following theorem holds. 
Theorem 4.1 ('spectral gap'). In the limit of small noise, the following asymptotics holds:

$$
\begin{aligned}
& \lambda_{0}=\lambda_{0}(\varepsilon)=0, \text { and } \Psi_{0}=e^{-\frac{2 U_{1}}{\varepsilon}} \\
& \lambda_{1}=\lambda_{1}(\varepsilon)=\frac{1}{2 \pi} \sqrt{U_{1}^{\prime \prime}(1)\left|U_{1}^{\prime \prime}(0)\right|} \cdot e^{-v / \varepsilon}(1+\mathcal{O}(\varepsilon)), \\
& \lambda_{2}=\lambda_{2}(\varepsilon) \geq C>0 \text { uniformly in } \varepsilon .
\end{aligned}
$$

The result of Theorem 4.1 plays a crucial role in our analysis. There is a spectral gap between the first eigenvalue and the rest of the spectrum. Consequently, only the first two terms of (4.2) can have an essential contribution to the SPA coefficient $\eta^{X}$.

\section{Asymptotics of the SPA coefficient}

The following theorem gives the asymptotics of the first two Fourier coefficients $a_{0}$ and $a_{1}$.

\section{Theorem 5.1.}

$$
\begin{aligned}
& a_{0}=\left\|\Psi_{0}\right\|, \\
& a_{1}=\frac{\left\|\Psi_{1}\right\|}{\left\|\Psi_{0}\right\|^{2}} \cdot \frac{\left\langle\Psi_{0}(-\cdot), \Psi_{1}\right\rangle}{\left\|\Psi_{1}\right\|^{2}-e^{-\frac{1}{2} T \lambda_{1}}\left\langle\Psi_{1}(-\cdot), \Psi_{1}\right\rangle}+r
\end{aligned}
$$

where $r$ vanishes in the limit of small noise and for $T \geq \exp \{(v+\delta) / \varepsilon\}, \delta$ being positive and sufficiently small.

Recall the definition (3.1) of the SPA coefficient. Denote

$$
S^{X}(\varepsilon, T)=\int_{0}^{\frac{1}{2}} \mathbf{E}_{\mu} X_{T s}^{\varepsilon, T} \cdot e^{2 \pi i s} d s .
$$

Then we identify $\eta^{X}=4\left|S^{X}\right|^{2}$.

Theorem 5.2. Let $T \geq \exp \{(v+\delta) / \varepsilon\}$ for $\delta$ positive and sufficiently small. Then the following expansion for $S^{X}$ holds in the small noise limit $\varepsilon \rightarrow 0$

$$
S^{X}=\frac{1}{\pi i} b_{0}+\frac{1}{\pi i-\frac{1}{2} \lambda_{1} T} b_{1}+r_{1}
$$

where the rest term $r_{1}$ tends to zero and the coefficients are given by

$$
\begin{aligned}
& b_{0}=\frac{\int y e^{-\frac{2 U_{1}(y)}{\varepsilon}} d y}{\int e^{-\frac{2 U_{1}(y)}{\varepsilon}} d y} \\
& b_{1}=-\frac{1+e^{-\frac{1}{2} T \lambda_{1}}}{2} \cdot \frac{\int y \Psi_{1}(y) d y}{\int e^{-\frac{2 U_{1}(y)}{\varepsilon}} d y} \cdot \frac{\left\langle\Psi_{0}(-\cdot), \Psi_{1}\right\rangle}{\left\|\Psi_{1}\right\|^{2}-e^{-\frac{1}{2} T \lambda_{1}}\left\langle\Psi_{1}(-\cdot), \Psi_{1}\right\rangle} .
\end{aligned}
$$


Finally,

$$
\eta^{X}=b_{0}^{2} \frac{4}{\pi^{2}} \frac{\left(\lambda_{1} T\right)^{2}}{4 \pi^{2}+\left(\lambda_{1} T\right)^{2}}+R .
$$

where $R$ tends to zero with $\varepsilon$.

Let us now study the resonance behaviour of the SPA coefficient $\eta^{X}$, i.e. investigate whether it has a local maximum in $\varepsilon$. We formulate the following Lemma which is obtained by application of Laplace's method of asymptotic expansions of singular integrals, see $[7,16]$ or also $[17,13]$.

Lemma 5.3 ('Laplace's method'). In the small noise limit, the following holds true:

$$
\begin{aligned}
& b_{0}=-1-\frac{1}{4} \frac{U_{1}^{(3)}(-1)}{U_{1}^{\prime \prime}(-1)^{2}} \varepsilon+\mathcal{O}\left(\varepsilon^{2}\right), \\
& b_{1}=-1+\mathcal{O}(\varepsilon),
\end{aligned}
$$

and consequently

$$
\begin{aligned}
& b_{0}^{2}=1+\frac{1}{2} \frac{U_{1}^{(3)}(-1)}{U_{1}^{\prime \prime}(-1)^{2}} \varepsilon+\mathcal{O}\left(\varepsilon^{2}\right), \\
& \left(b_{0}-b_{1}\right)^{2}=\mathcal{O}\left(\varepsilon^{2}\right) .
\end{aligned}
$$

Now we can formulate our main theorem.

Theorem 5.4. Let us fix $\delta$ positive and sufficiently small and $\Delta>v+\delta$. Let also $U_{1}(x)-2 U_{1}(-x)<v+V$ for all $x \in \mathbb{R}$ (no strong asymmetry!). Then for $T \rightarrow \infty$ and $\varepsilon$ from the domain

$$
\frac{v+\delta}{\log T} \leq \varepsilon \leq \frac{\Delta}{\log T}
$$

the following asymptotic expansion for the SPA coefficient holds:

$$
\eta^{X}(\varepsilon, T)=\frac{4}{\pi^{2}}\left(1+\frac{1}{2} \frac{U_{1}^{(3)}(-1)}{U_{1}^{\prime \prime}(-1)^{2}} \varepsilon\right)+\mathcal{O}\left(\frac{1}{\log ^{2} T}\right) .
$$

This result has the following surprising consequences.

Corollary 5.5. For $T \rightarrow \infty$ and $\varepsilon \in\left[\frac{v+\delta}{\log T}, \frac{\Delta}{\log T}\right]$ the SPA coefficient is a decreasing function of $\varepsilon$ if $U_{1}^{(3)}(-1)<0$ and an increasing function of $\varepsilon$ if $U_{1}^{(3)}(-1)>0$.

Thus, the SPA coefficient as quality measure for tuning shows no resonance in a domain above Freidlin's threshold for quasi-deterministic periodicity (Theorem 2.2). This contradicts the physical intuition for the 'effective dynamics'. The reason for this surprising phenomenon can only be hidden in the intrawell behaviour of the diffusion neglected when passing to the reduced Markov chain. We return to this question later. Let us next study mathematically the 'effective dynamics' of the diffusion (1.4). 


\section{The 'effective dynamics': two-state Markov chain}

The idea of approximation of diffusions in potential landscapes by appropriate finite state Markov chains in the context of stochastic resonance was suggested by Eckmann and Thomas [6], and C. Nicolis [15], and developed by McNamara and Wiesenfeld [14]. In this section we follow [17, 13]. The discrete time case was studied in [12].

In order to catch the main features of the interwell hoppings of the diffusion (1.4) we consider the time inhomogeneous Markov chain $Y^{\varepsilon, T}$ living on the diffusion's meta-stable states \pm 1 . The infinitesimal generator of $Y^{\varepsilon, T}$ is periodic in time and is given by

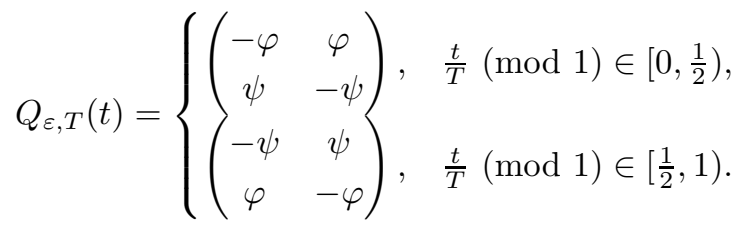

The transition rates $\varphi$ and $\psi$ which are responsible for the similarity of the two processes are chosen to be exponentially small in $\varepsilon$ :

$$
\varphi=\frac{1}{2 \pi} \sqrt{U_{1}^{\prime \prime}(-1)\left|U_{1}^{\prime \prime}(0)\right|} \cdot e^{-V / \varepsilon} \text { and } \psi=\frac{1}{2 \pi} \sqrt{U_{1}^{\prime \prime}(1)\left|U_{1}^{\prime \prime}(0)\right|} \cdot e^{-v / \varepsilon} .
$$

To exponential order they correspond (as they should) to the inverses of the Kramers' transition times (see Theorem 2.1). The invariant measure of $Y_{T t}^{\varepsilon, T}$ can be obtained as a solution of a forward Kolmogorov equation and is given by

$$
\begin{aligned}
& \nu^{-}(t)=\frac{\psi}{\varphi+\psi}+\frac{\varphi-\psi}{\varphi+\psi} \frac{e^{-(\varphi+\psi) T t}}{1+e^{-\frac{1}{2}(\varphi+\psi) T t}}, \\
& \nu^{+}(t)=\frac{\varphi}{\varphi+\psi}-\frac{\varphi-\psi}{\varphi+\psi} \frac{e^{-(\varphi+\psi) T t}}{1+e^{-\frac{1}{2}(\varphi+\psi) T t}}, \quad t \in\left[0, \frac{1}{2}\right],
\end{aligned}
$$

and $\nu^{ \pm}(t)=\nu^{\mp}\left(t+\frac{1}{2}\right)$ for $t \geq 0$.

We define the SPA coefficient $\eta^{Y}$ for the Markov chain $Y^{\varepsilon, T}$ analogously to (3.1). In the much simpler setting given it can be found explicitly.

Theorem 6.1. For all $\varepsilon>0$ and $T>0$ the following holds:

$$
\eta^{Y}(\varepsilon, T)=\frac{4}{\pi^{2}} \frac{T^{2}(\varphi-\psi)^{2}}{4 \pi^{2}+T^{2}(\varphi+\psi)^{2}} .
$$

Compare (6.1) with (5.2). Since $(\varphi \pm \psi)^{2} \approx \lambda_{1}^{2}$ in the limit of small $\varepsilon$, the formulae for $\eta^{X}$ and $\eta^{Y}$ differ only in the 'geometric' pre-factor $b_{0}^{2}$ and the asymptotically negligible rest term $R$.

The exact formula (6.1) allows to study the local maxima of $\eta^{Y}$ as a function of noise intensity for large periods $T$. 
Theorem 6.2. In the limit $T \rightarrow \infty$ the function $\varepsilon \mapsto \eta^{Y}(\varepsilon, T)$ has a local maximum at

$$
\varepsilon(T) \approx \frac{v+V}{2} \frac{1}{\log T} .
$$

The 'resonance' behaviours of $\eta^{X}$ and $\eta^{Y}$ are quite different. Whereas the diffusion's SPA has no extremum for small $\varepsilon$, the Markov chain's always has. What can be responsible for this discrepancy? Note that the Markov chain mimicks only the interwell dynamics of the diffusion. Thus, the SPA coefficient $\eta^{Y}$ measures only the spectral energy contributed by interwell jumps. On the other hand, $\eta^{X}$ also counts the numerous intrawell fluctuations of the diffusion. These fluctuations have small energy. But since the diffusion spends most of its time near \pm 1 the local asymmetries of the potential at these points become important and destroy optimal tuning.

To underpin this heuristics mathematically, let us now make the idea of neglecting the diffusion's intrawell fluctuations precise. For example, we cut off those among them which have not enough energy to reach half the height of the potential barrier between the wells. Consider the cut-off function $g$ defined by

$$
g(x)= \begin{cases}-1, & x \in\left[x_{1}, x_{2}\right] \\ 1, & x \in\left[y_{1}, y_{2}\right] \\ x, & \text { otherwise }\end{cases}
$$

where $x_{1}<-1<x_{2}<0$ and $0<y_{1}<1<y_{2}$ are such that $U_{1}\left(x_{1}\right)=U_{1}\left(x_{2}\right)=$ $-\frac{V}{4}$ and $U_{1}\left(y_{1}\right)=U_{1}\left(y_{2}\right)=-\frac{v}{4}$, see Fig. 6 . Now we study the modified SPA

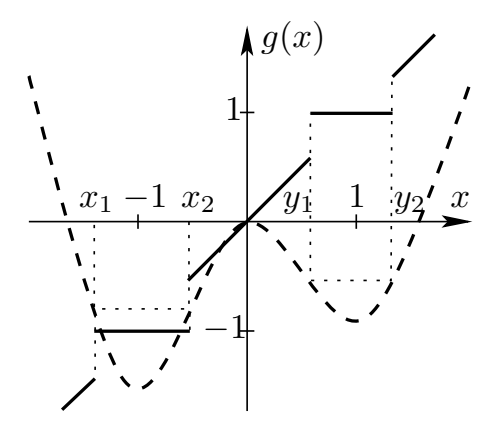

Fig. 6. Function $g$ designed to cut off diffusion's intrawell dynamics.

coefficient of a diffusion defined by

$$
\widetilde{\eta}^{X}(\varepsilon, T)=\left|\int_{0}^{1} \mathbf{E}_{\mu}\left[g\left(X_{T s}^{\varepsilon, T}\right)\right] e^{2 \pi i s} d s\right|^{2} .
$$


Following the steps of Section 5 we obtain a formula for $\widetilde{\eta}^{X}$ which is quite similar to (5.2) and (6.1):

$$
\widetilde{\eta}^{X}(\varepsilon, T)=\widetilde{b}_{0}^{2} \frac{4}{\pi^{2}} \frac{\left(\lambda_{1} T\right)^{2}}{4 \pi^{2}+\left(\lambda_{1} T\right)^{2}}+\widetilde{R}
$$

where $\widetilde{R}$ is a small rest term, and

$$
\widetilde{b}_{0}^{2}=\left(\frac{\int g(y) e^{-\frac{2 U_{1}(y)}{\varepsilon}} d y}{\int e^{-\frac{2 U_{1}(y)}{\varepsilon}} d y}\right)^{2}=1-4 \sqrt{\frac{U_{1}^{\prime \prime}(-1)}{U_{1}^{\prime \prime}(1)}} e^{-\frac{V-v}{\varepsilon}}(1+\mathcal{O}(\varepsilon))
$$

(compare to $(5.3))$.

The modified geometric pre-factor $\widetilde{b}_{0}^{2}$ is essentially smaller than its counterpart $b_{0}^{2}$. This has crucial influence on the SPA coefficient $\widetilde{\eta}^{X}$ : in the limit of large period and small noise its behaviour now reminds of $\eta^{Y}$.

Theorem 6.3. Let the assumptions of Theorem 5.4 hold. Then for any $\gamma>1$ in the limit $T \rightarrow \infty$ the function $\varepsilon \mapsto \widetilde{\eta}^{X}(\varepsilon, T)$ has a local maximum on

$$
\left[\frac{1}{\gamma} \frac{v+V}{2} \frac{1}{\log T}, \gamma \frac{v+V}{2} \frac{1}{\log T}\right]
$$

In other words, the optimal tuning for the measure of goodness $\widetilde{\eta}^{X}$ exists and is given approximately by

$$
\varepsilon(T) \approx \frac{v+V}{2} \frac{1}{\log T}
$$

\section{References}

[1] V. S. Anishchenko, A. B. Neiman, F. Moss, and L. Schimansky-Geier. Stochastic resonance: noise-enhanced order. Physics-Uspekhi, 42(1):7-36, 1999.

[2] R. Benzi, G. Parisi, A. Sutera, and A. Vulpiani. The mechanism of stochastic resonance. J. Phys. A, 14:453-457, 1981.

[3] R. Benzi, G. Parisi, A. Sutera, and A. Vulpiani. Stochastic resonance in climatic changes. Tellus, 34:10-16, 1982.

[4] R. Benzi, G. Parisi, A. Sutera, and A. Vulpiani. A theory of stochastic resonance in climatic change. SIAM J. Appl. Math., 43:563-578, 1983.

[5] M. I. Budyko. The effect of solar radiation variations on the climate of the earth. Tellus, 21:611-619, 1969.

[6] J.-P. Eckmann and L. E. Thomas. Remarks on stochastic resonance. J. Phys. A, 15:261-266, 1982.

[7] A. Erdélyi. Asymptotic expansions. Dover Publications, Inc., New York, 1956.

[8] M. I. Freidlin. Quasi-deterministic approximation, metastability and stochastic resonance. Physica D, 137(3-4):333-352, 2000.

[9] M. I. Freidlin and A. D. Wentzell. Random perturbations of dynamical systems, volume 260 of Grundlehren der Mathematischen Wissenschaften. Springer, New York, NY, second edition, 1998. 
[10] L. Gammaitoni, P. Hänggi, P. Jung, and F. Marchesoni. Stochastic resonance. Reviews of Modern Physics, 70:223-287, January 1998.

[11] P. Imkeller. Energy balance models - viewed from stochastic dynamics. In Imkeller, P. et al., editors, Stochastic climate models. Proceedings of a workshop, Chorin, Germany, Summer 1999., volume 49 of Prog. Probab., pages 213-240, Basel, 2001. Birkhäuser.

[12] P. Imkeller and I. Pavlyukevich. Stochastic resonance in two-state Markov chains. Arch. Math., 77(1):107-115, 2001.

[13] P. Imkeller and I. Pavlyukevich. Model reduction and stochastic resonance. Stochastics and Dynamics, 4, 2002.

[14] B. McNamara and K. Wiesenfeld. Theory of stochastic resonance. Physical Review A (General Physics), 39:4854-4869, May 1989.

[15] C. Nicolis. Stochastic aspects of climatic transitions - responses to periodic forcing. Tellus, 34:1-9, 1982.

[16] F. W. J. Olver. Asymptotics and special functions. Computer Science and Applied Mathematics. Academic Press, a subsidiary of Harcourt Brace Jovanovich, Publishers, New York - London, 1974.

[17] I. E. Pavlyukevich. Stochastic Resonance. PhD thesis, Humboldt-Universität, Berlin, 2002. Logos-Verlag, ISBN 3-89722-960-9.

[18] W. B. Sellers. A global climate model based on the energy balance of the earthatmosphere system. J. Appl. Meteor., 8:301-320, 1969.

[19] K. Wiesenfeld and F. Jaramillo. Minireview of stocastic resonance. Chaos, 8:539-548, September 1998.

Institut für Mathematik, Humboldt-Universität zu Berlin, Unter den Linden 6, 10099 Berlin, Germany

E-mail address: imkeller@mathematik.hu-berlin.de

Institut für Mathematik, Technische Universität Berlin, Straße des 17. Juni 136, 10623 Berlin, Germany

E-mail address: pavljuke@math.tu-berlin.de 